$\mathrm{p}<0.05$ ). In the post-dilation group, however, post-procedural plasma BNP levels were significantly increased when compared with the pre-procedural level $((70.3 \pm 48.2) \mathrm{ng} / \mathrm{l}$ vs $(55.1 \pm 35.4) \mathrm{ng} /$ $1, \mathrm{p}<0.05)$. Pre-procedural plasma BNP levels were similar between the two groups $(p>0.05)$. However, post-procedural plasma BNP levels in the post-dilation group were also significantly higher than those in the conventional group $(p<0.05)$.

Conclusions High-pressure post-dilation following coronary stent deployment resulted in a significant increase of plasma BNP levels, which may be related to the deterioration of cardiac function shortly after the interventional procedure.

\section{GW23-e0158 CHANGES OF PLASIMA B-TYPE NATRIURETIC PEPTIDE LEVELS AFTER HIGH-PRESSURE POST-DILATION FOLLOWING CORONARY STENT DEPLOYMENT}

\author{
doi:10.1136/heartjnl-2012-302920l.19
}

Gang-Yong Wu, Gang-Jun Zong. Department of Cardiology, 101 Hospital of PLA

Objectives To evaluate the changes of plasma B-type natriuretic peptide (BNP) levels after high-pressure post-dilation following coronary stent deployment.

Methods From June 2007 to June 2010, a total of 450 patients underwent PCI in our hospital. After excluding those with acute myocardial infarction, chronic occlusive lesions, major complications such as coronary perforation, dissection, major bleeding, and contrast-induced nephropathy during or after the original interventional procedures. A total of 143 patients undergoing percutaneous coronary intervention for single-vessel disease of the left anterior descending artery were enrolled into the study and retrospectively analysed. All interventional procedures for the enrolled patients were performed by same interventional cardiologists. All patients were divided into two groups: a conventional-pressure stent deployment group (conventional group) and a non-compliant balloon high-pressure post-dilation group (post-dilation group). General information, coronary stent parameters and imaging data in both groups were collected and analysed. The plasma BNP and troponin I (TnI) levels immediately before and $24 \mathrm{~h}$ after the interventional procedures were compared between the two groups.

Results There were no significant differences between the two groups in terms of general information, coronary stent parameters, as well as pre- and post-procedural TnI levels (all $p>0.05$ ). And, in the post-dilaiton group, the mean post-dilation pressure was $(20.4 \pm 3.9) \mathrm{KPa}$. In the conventional group, post-procedural plasma BNP levels were significantly reduced when compared with the pre-procedural levels $((34.7 \pm 17.7) \mathrm{ng} / \mathrm{l}$ vs $(43.3 \pm 19.7) \mathrm{ng} / \mathrm{l}$, 